\title{
ARTICLE
}

Received 19 Jul 2016 | Accepted 23 Aug 2016 | Published 26 Sep 2016

DOl: $10.1038 /$ ncomms12978

OPEN

\section{Atomically thin quantum light-emitting diodes}

\author{
Carmen Palacios-Berraquero ${ }^{1, \star}$, Matteo Barbone ${ }^{2, \star}$, Dhiren M. Kara ${ }^{1}$, Xiaolong Chen², llya Goykhman², \\ Duhee Yoon ${ }^{2}$, Anna K. Ott ${ }^{2}$, Jan Beitner ${ }^{1}$, Kenji Watanabe ${ }^{3}$, Takashi Taniguchi ${ }^{3}$, Andrea C. Ferrari ${ }^{2}$ \\ $\&$ Mete Atatüre ${ }^{1}$
}

Transition metal dichalcogenides are optically active, layered materials promising for fast optoelectronics and on-chip photonics. We demonstrate electrically driven single-photon emission from localized sites in tungsten diselenide and tungsten disulphide. To achieve this, we fabricate a light-emitting diode structure comprising single-layer graphene, thin hexagonal boron nitride and transition metal dichalcogenide mono- and bi-layers. Photon correlation measurements are used to confirm the single-photon nature of the spectrally sharp emission. These results present the transition metal dichalcogenide family as a platform for hybrid, broadband, atomically precise quantum photonics devices.

\footnotetext{
${ }^{1}$ Cavendish Laboratory, University of Cambridge, J.J. Thomson Ave., Cambridge CB3 OHE, UK. ${ }^{2}$ Cambridge Graphene Centre, University of Cambridge, Cambridge CB3 OFA, UK. ${ }^{3}$ Advanced Materials Laboratory, National Institute for Materials Science, Tsukuba, Ibaraki 305-0034, Japan. * These authors contributed equally to this work. Correspondence and requests for materials should be addressed to A.C.F. (email: acf26@cam.ac.uk) or to M.A. (email: ma424@cam.ac.uk).
} 
ncorporating single-photon sources into optoelectronic circuits is a key challenge to develop scalable quantumphotonic technologies. Despite a plethora of single-photon sources reported to-date, all-electrical operation, desired for systems integration, is reported for only a few ${ }^{1-4}$. Layered materials (LMs) offer novel opportunities for next-generation photonic and optoelectronic technologies ${ }^{5,6}$, such as lasers ${ }^{7,8}$, modulators $^{9,10}$ and photodetectors ${ }^{11}$, and are compatible with the silicon platform ${ }^{12}$.

The attractiveness of single-photon sources in $\mathrm{LMs}^{13-18}$ stems from their ability to operate at the fundamental limit of few-atom thickness, providing the potential to integrate into conventional and scalable high-speed optoelectronic systems ${ }^{19,20}$. Transition metal dichalcogenides (TMDs), being optically active layered semiconductors, are particularly suitable for developing quantum-light generating devices.

Here we demonstrate that LMs enable all-electrical singlephoton generation over a broad spectrum. We use a lightemitting diode (LED) realized by vertical stacking of LMs and achieve charge injection into the active layer containing quantum emitters. We show that quantum emitters in tungsten diselenide $\left(\mathrm{WSe}_{2}\right)^{13-17}$ can operate electrically. We further report all-electrical single-photon generation in the visible spectrum from a new class of quantum emitters in tungsten disulfide $\left(\mathrm{WS}_{2}\right)$. Our results highlight the promise of LMs as a new platform for broadband hybrid all-integrated quantum-photonic circuits.

\section{Results}

Design and operation of a vertical TMD-based LED. We realize an LED based on a single tunnelling junction made of vertically stacked LMs (see 'Methods', Supplementary Figs 1-8 and Supplementary Notes 1-3). Figure la is a typical optical microscope image of such a device. From bottom to top, three layers form a heterostructure on a silicon/silicon dioxide $\left(\mathrm{Si} / \mathrm{SiO}_{2}\right)$ substrate: a single layer of graphene (SLG), a thin (2-6 atomic layers) sheet of hexagonal boron nitride $(\mathrm{hBN})$ and a mono- or bi-layer of TMD, such as $\mathrm{WSe}_{2}$. The $\mathrm{WSe}_{2}$, exfoliated from a naturally p-doped bulk crystal, is the optically active layer hosting single-photon sources. Metal electrodes provide electrical contact to the SLG and TMD layers. To obtain electroluminescence (EL), we inject electrons from the SLG to the p-doped $\mathrm{WSe}_{2}$ through the $\mathrm{hBN}$ tunnel barrier (see Supplementary Fig. 9 for current-voltage characteristics of the devices). A vertically stacked heterojunction allows for EL from the whole device area, unlike lateral Schottky junction or split-gate $\mathrm{p}-\mathrm{n}$ junction devices ${ }^{21-23}$, and provides the benefit of atomically precise interfaces and barrier thicknesses ${ }^{24}$. We leave the optically active TMD layer exposed at the top of the device purposefully to offer interfacing with other systems.

Figure $1 \mathrm{~b}$ illustrates the operational concept of our LED. At zero bias between the SLG and the monolayer TMD contacts, the Fermi energy $\left(E_{\mathrm{F}}\right)$ of the system is constant across the heterojunction, preventing net charge flow (current) between the layers (Fig. 1b, top). A negative bias applied to the SLG electrode raises the SLG $E_{\mathrm{F}}$ above the minimum of the conduction band $\left(E_{\mathrm{C}}\right)$ of grounded $\mathrm{WSe}_{2}$. This induces electrons to tunnel from the SLG into the monolayer $\mathrm{WSe}_{2}$. This initiates photoemission through radiative recombination between the tunnelled electrons and the holes residing in the optically active $\mathrm{WSe}_{2}$ area (Fig. 1b, bottom). Figure 1c compares the EL and photoluminescence (PL) spectra from this monolayer-WSe ${ }_{2}$-based LED device for two operation temperatures, room temperature (RT) and $10 \mathrm{~K}$ (see 'Methods' and Supplementary Fig. 10 for measurement setup). PL at RT is given by the black curve in the lower panel with a a

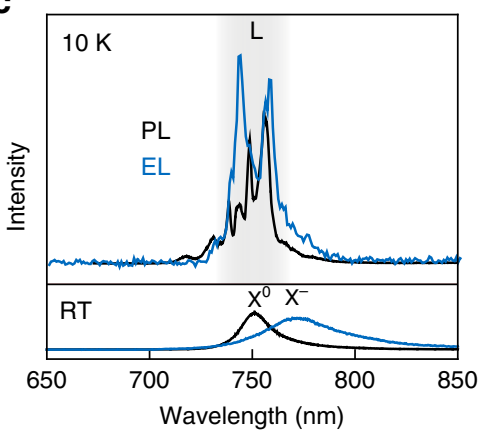

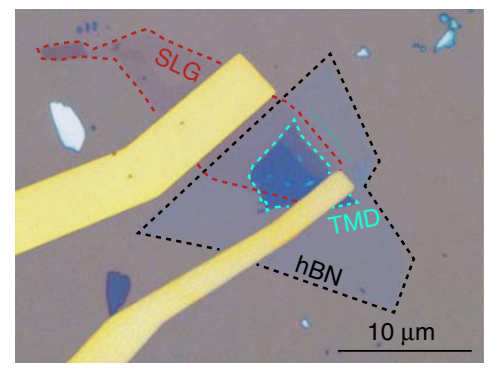

b
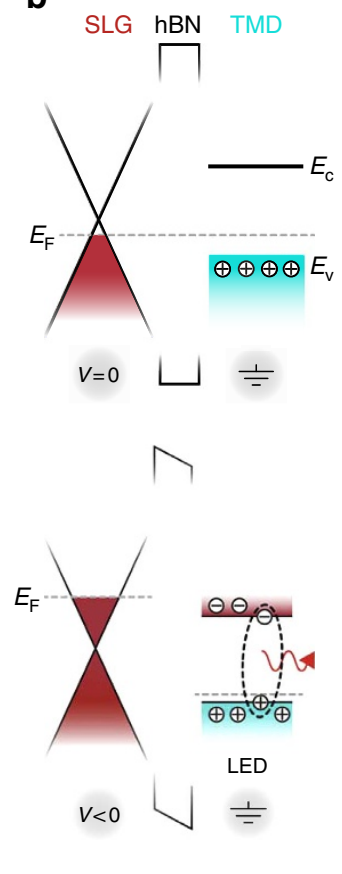

Figure 1 | Design and operation of a TMD-based LED. (a) Optical microscope image of a typical device used in our experiments. The dotted lines highlight the footprint of the SLG, hBN and the TMD layers individually. The $\mathrm{Cr} / \mathrm{Au}$ electrodes contact the SLG and TMD layers to provide an electrical bias. (b) Heterostructure band diagram. The top illustration shows the case for zero-applied bias and the bottom illustration shows the case for a finite negative bias applied to the SLG. Tuning the SLG Fermi level $\left(E_{\mathrm{F}}\right)$ across the TMD conduction band edge $\left(E_{C}\right)$ allows electron tunnelling from the SLG to the TMD, resulting in light emission via radiative recombination of the electrons with the holes residing in the $\mathrm{p}$-doped TMD layer. The appearance of valence-band holes below the Fermi level is due to the natural p-doping of $\mathrm{WSe}_{2}$. (c) An example of layered LED emission spectra for an optically active layer of $\mathrm{WSe}_{2}$. Top (bottom) spectra correspond to $10 \mathrm{~K}(\mathrm{RT})$ operation temperature, where the black and blue spectra are obtained by optical excitation and electrical excitation, respectively.

broad peak at $750 \mathrm{~nm}$ corresponding to the monolayer $\mathrm{WSe}_{2}$ unbound neutral exciton emission, $\mathrm{X}^{8}$ (ref. 25). Under electrical excitation the main peak is shifted $\sim 20 \mathrm{~nm}$ to longer wavelengths, which is commensurate with the charged exciton, $\mathrm{X}^{-}$(ref. 26), as shown in the blue curve. The black and blue spectra in the upper panel of Fig. 1c show the device's $\mathrm{PL}$ and EL emission at $10 \mathrm{~K}$, respectively. Because of the increased bandgap at low temperatures, the unbound exciton emission is shifted to shorter wavelengths by $\sim 30 \mathrm{~nm}$ (ref. 27). Consistent with recent reports ${ }^{13-17,27}$, extra structure arising from localized exciton state emission (L) appears at longer wavelengths in the PL spectrum. Critically, these features are also visible under electrical excitation. In the low-current regime ( $<1 \mu \mathrm{A}$ for this device) they dominate the EL spectrum, as shown in Fig. 1c, indicating that localized exciton states respond more efficiently to charge injection than the delocalized ones.

Electrically driven quantum light in a WSe $e_{2}$-based LED. Figure $2 \mathrm{a}$ is a spatial map of integrated EL from a $\mathrm{WSe}_{2}$-based LED device at $10 \mathrm{~K}$. The active region of this device consists of adjacent monolayer and bilayer active areas, both in contact 
a

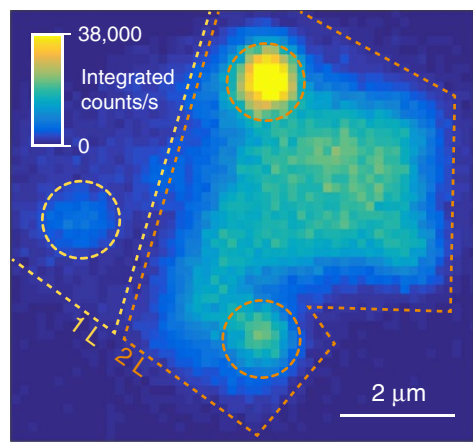

b

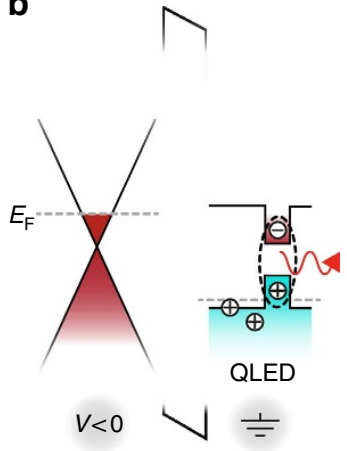

C

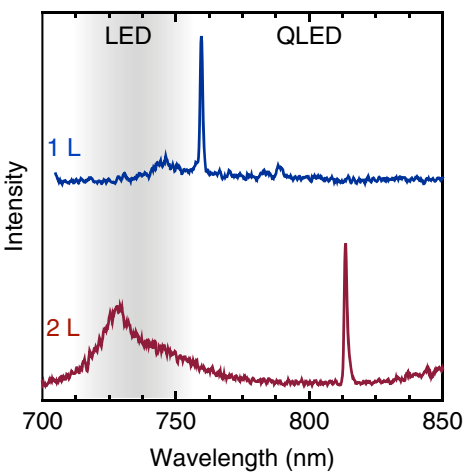

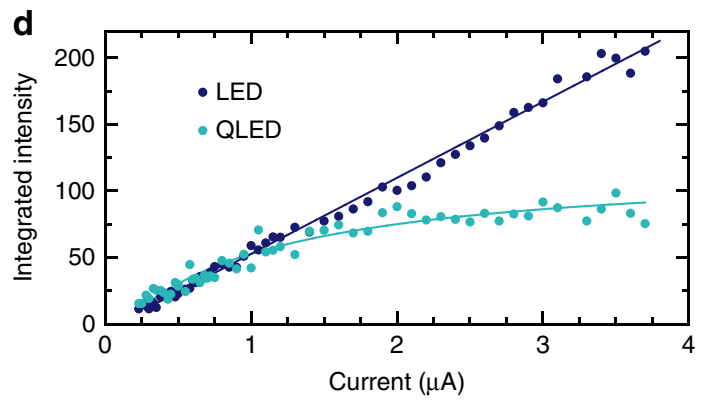

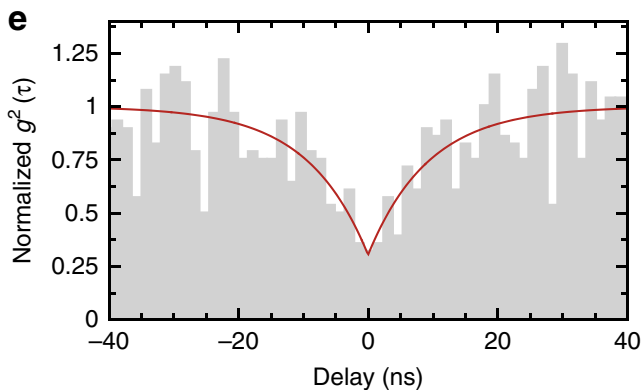

Figure 2 | WSe $\mathbf{~}_{2}$-based QLED operation in the near infrared spectrum. (a) A raster-scan map of integrated EL intensity from monolayer and bilayer $\mathrm{WSe}_{2}$ areas of the QLED for an injection current of $3 \mu \mathrm{A}(12.4 \mathrm{~V})$. The dotted circles highlight the submicron localized emission in this device. (b) A schematic energy band diagram, similar to that in Fig. 1b, including the confined electronic states of the quantum dots. EL emission from quantum dots typically starts at lower bias than the conventional LED operation threshold. (c) Typical EL emission spectra for quantum dots in the monolayer (top) and bilayer (bottom) $\mathrm{WSe}_{2}$. The shaded area highlights the spectral window for LED emission due to bulk WSe $\mathrm{W}_{2}$ excitons, whereas QLED operation produces spectra at longer wavelengths. (d) Comparison of the integrated EL intensity for the WSe $\mathrm{Clayer}_{2}$ and for a quantum dot as a function of the applied current. The linear increase in WSe 2 layer EL contrasts with the saturation behaviour of the QLED emission. (e) Intensity-correlation function, $g^{(2)}(\tau)$, for the same emitter displaying the antibunched nature of the EL signal, $g^{(2)}(0)=0.29 \pm 0.08$, and a rise-time of $9.4 \pm 2.8 \mathrm{~ns}$.

with the ground electrode. The brighter area in Fig. 2a corresponds to the bilayer, suggesting that most of the injected current flows through this region (see Supplementary Fig. 11). In addition to the spatially uniform light emission from delocalized excitons, we observe quantum LED (QLED) operation in the form of highly localized light emission from both the monolayer and the bilayer $\mathrm{WSe}_{2}$, identified by the dotted circles (Fig. 2a). These localized states lie within the bandgap of $\mathrm{WSe}_{2}$, and therefore emit at lower energies (longer wavelength) with respect to the bulk exciton emission (see Fig. $2 \mathrm{~b}$ ) ${ }^{13-17}$. Figure 2c shows example emission spectra from these sites, where the top (bottom) spectrum belongs to a quantum emitter in the monolayer (bilayer) $\mathrm{WSe}_{2}$. We observe spectrally isolated peaks from multiple locations in most devices with linewidths ranging between 0.8 and $3 \mathrm{~nm}$. We see on average 1-2 emitters per $\sim 40 \mu \mathrm{m}^{2}$ active device area. Electrically excited narrow lines coming from bilayer $\mathrm{WSe}_{2}$ regions are typically redshifted with respect to those coming from the monolayer regions ${ }^{28}$. The emission peaks of Fig. $2 \mathrm{c}$ are unpolarized, and the fine structure splitting reported in PL experiments $(\sim 0.3 \mathrm{~nm}$ (refs 13-17)) is not resolvable due to the broader linewidths we observe in EL. On the timescale of seconds, most emitters show spectral wandering, of up to $2 \mathrm{~nm}$, similar to that seen in our PL measurements. Gating and encapsulation of the active layer should aid in reducing the broad linewidths observed here, which we attribute to charge noise in the device. Slow spectral fluctuations can further be reduced through active feedback, for example via the direct current Stark shift ${ }^{29,30}$. A fraction of the quantum emitters display blinking, discrete spectral jumps or multiple spectral lines at similar timescales (see Supplementary Fig. 12). We also see that, as in PL, the electrically driven emitters display robust operation, withstanding multiple (3-5) cooling/ heating cycles and several hours of measurement under uninterrupted current flow.

Figure $2 \mathrm{~d}$ plots the current dependence of the integrated EL intensity from a quantum emitter, as well as from the unbound monolayer $\mathrm{WSe}_{2}$ excitons. The latter shows a predominantly linear relation between emission intensity and injected current; however, EL emission from the quantum emitter shows clear saturation as a function of current, a universal behaviour seen with single-photon sources ${ }^{31}$ (see Supplementary Fig. 13 for a plot of the unbound exciton and quantum emitter spectra as a function of current). Figure 2e shows the measured intensitycorrelation function, $g^{(2)}(\tau)$, of the integrated EL emission from a $\mathrm{WSe}_{2}$-based QLED using a Hanbury Brown and Twiss interferometer (see 'Methods'). The value of the normalized $g^{(2)}(0)$ drops to $0.29 \pm 0.08$, well below the threshold value of 0.5 , expected for a single-photon source ${ }^{1}$. We note that these data are not corrected for background emission within the broad spectral window of detection or for the photon-counting detector dark counts, which together contribute to the non-zero value of $g^{(2)}(0)$. While our results manifest the single-photon nature of the electrically generated emission as proof-of-concept, the immediate usability of these quantum emitters as single-photon sources would benefit from spectral filtering to suppress the background emission. Further technical improvements, such as 
optimized designs for charge injection, may be possible once the nature of these emitters is identified.

All-electrical generation of single-photon emission in $\mathrm{WS}_{2}$. In TMD-based quantum emitters, the host material influences the quantized energy levels and consequently their emission wavelength. Therefore, in order to obtain single-photon emission in a complementary part of the spectrum, we replace the monolayer of $\mathrm{WSe}_{2}$ with $\mathrm{WS}_{2}$ (exfoliated from an n-doped bulk crystal) as the active layer; the rest of the QLED device structure remains unchanged. Figure 3a,b displays the spatial maps of integrated EL emission from a WS $\mathrm{WS}_{2}$-based QLED device at high- $(0.665 \mu \mathrm{A})$ and low- $(0.570 \mu \mathrm{A})$ current injection, respectively. At high current, the emission intensity is spatially uniform in the monolayer. At low currents, however, a spatially localized emission site dominates, indicating that $\mathrm{WS}_{2}$ also hosts localized quantum emitters. Figure $3 \mathrm{c}$ shows the EL spectrum as a function of injection current, demonstrating that the low current $(\sim 0.570 \mu \mathrm{A})$ regime leads to a narrow $(\sim 4 \mathrm{~nm})$ emission at $640 \mathrm{~nm}$, a line cut (in blue) of which is in the bottom right panel. Figure $3 c$ (upper right plot) also shows how the EL spectrum is broadened significantly when driven strongly at an injection current of $1.8 \mu \mathrm{A}$. The EL at $640 \mathrm{~nm}$ lies within the spectral region of an emission band that appears, in addition to the unbound exciton emission, at low temperature $(<10 \mathrm{~K})$ under optical excitation (see Supplementary Figs 14 and 15 for details and Supplementary Fig. 16 for a comparison between the raster-scan maps of integrated EL intensity from $\mathrm{WS}_{2}$ and $\mathrm{WSe}_{2}$ at RT and $10 \mathrm{~K})$. Operating in the low-current range ensures that the full EL spectrum is dominated by single-photon emission from the quantum emitter, obviating any need for tailored spectral filtering. The intensity-correlation measurement for EL in this regime, without spectral filtering, yields the $g^{(2)}(\tau)$ data in Fig. 3d. Similar to the $\mathrm{WSe}_{2}$ emitters, the uncorrected, but normalized, $g^{(2)}(0)$ falls below 0.5 to $0.31 \pm 0.05$, indicating that $\mathrm{WS}_{2}$ supports stable QLED operation, generating single photons in the visible spectral range.

\section{Discussion}

Our TMD-based QLEDs rely on a single tunnelling heterojunction design, where a wide range of TMDs can be active materials. Other designs, employing a back gate to tune $E_{\mathrm{F}}$ of the active TMD layer and providing electrostatic tuning of the EL emission spectrum, can enhance the versatility of these devices. One possibility is the deterministic control over the charging states of confined excitons ${ }^{32}$, en route to spin control $^{33}$ and entangled photon generation ${ }^{34}$. We also note that the emission wavelength range for $\mathrm{WSe}_{2}$ emitters can match rubidium transitions $(\sim 780 \mathrm{~nm})$ for exploring quantum storage possibilities. Similarly, silicon-vacancy centres $(\sim 737 \mathrm{~nm})$ and nitrogenvacancy centres $(\sim 637 \mathrm{~nm})$ in diamond can have matching transitions with the $\mathrm{WSe}_{2}$ and $\mathrm{WS}_{2}$ QLEDs, respectively, for interfacing hybrid quantum systems via distributed or on-chip photonic channels. Other TMDs are likely to yield similar results decorating different spectral windows. Our results demonstrate that LMs are a platform for fully integrable and atomically precise devices for quantum photonics technologies.
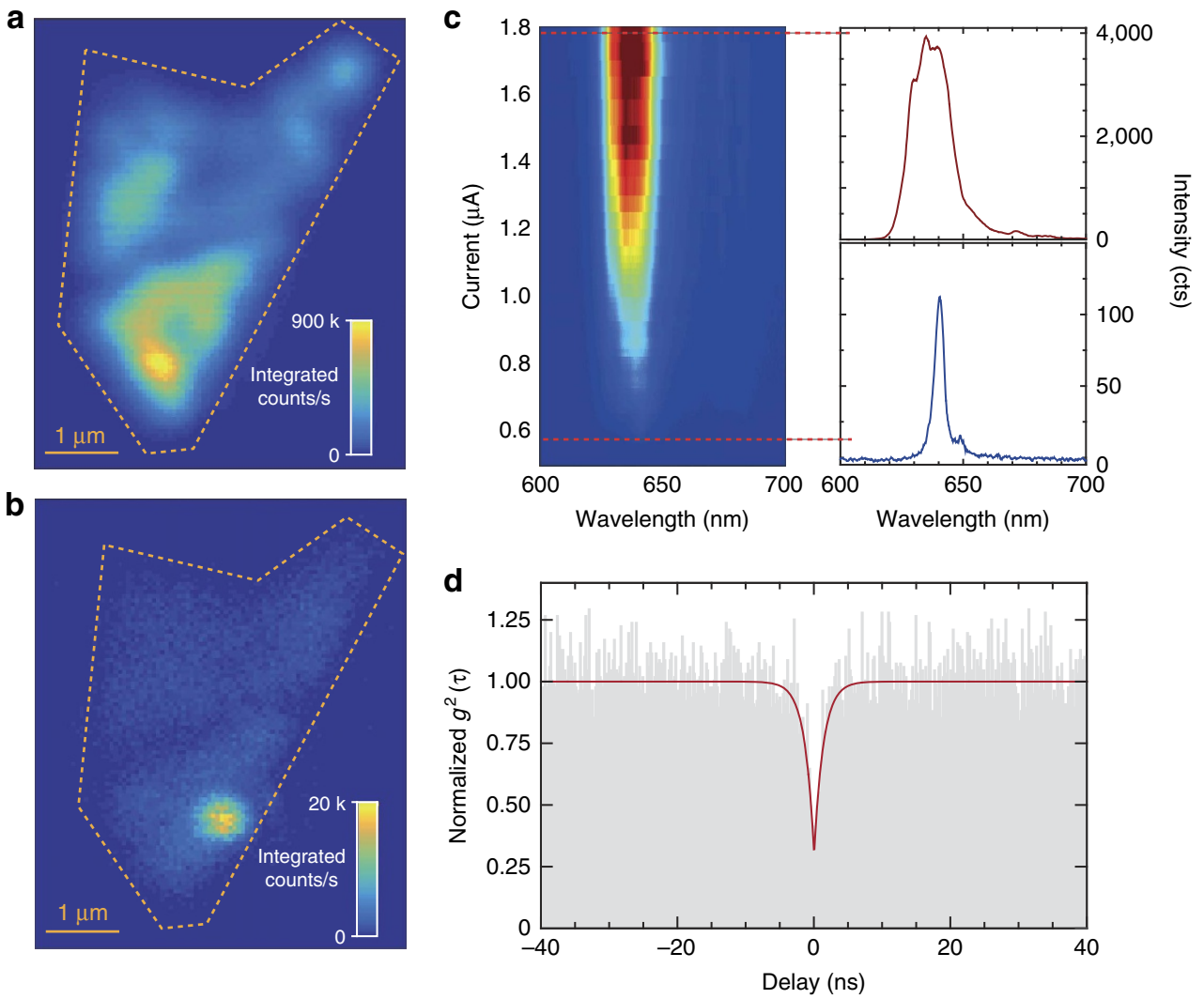

Figure 3 | WS $\mathbf{W}_{\mathbf{2}}$-based QLED operation in the visible spectrum. A raster-scan map of integrated EL intensity from the monolayer WS 2 area of the device: (a) at $0.665 \mu \mathrm{A}$ injection current (bias $2.08 \mathrm{~V}$ ), where the emission is delocalized and roughly uniform, and (b) at $0.570 \mu \mathrm{A}(1.97 \mathrm{~V}$ ), where the highly localized QLED emission dominates over the unbound $W_{2}$ exciton emission. (c) A map of the EL spectrum, displaying the evolution of the WS 2 QD emission spectrum as a function of current. The spectrum at the top (bottom) of the panel is a line cut for injection current of $1.8 \mu \mathrm{A}$ ( $0.578 \mu \mathrm{A}$ ). (d) Intensity-correlation function, $g^{(2)}(\tau)$, for the same quantum dot displaying the antibunched nature of the EL signal, $g^{(2)}(0)=0.31 \pm 0.05$, and a rise-time of $1.4 \pm 0.15 \mathrm{~ns}$. 


\section{Methods}

Device fabrication. We exfoliate the LMs on oxidized Si wafers by micromechanical cleavage of bulk crystals of graphite (NGS Naturgraphit), TMDs (HQ Graphene) and hBN single crystals, grown by the temperature-gradient method under high pressure and high temperature ${ }^{35}$. Mono-, bi- and few-layer samples are identified by a combination of optical contrast (see Supplementary Figs 1, 3 and 5$)^{36}$, Raman spectroscopy (see Supplementary Figs 2, 4a, 6 and $\left.7 a\right)^{37}$, PL (see Supplementary Figs $4 \mathrm{~b}$ and $7 \mathrm{~b}$ ) and atomic force microscopy (AFM) (see Supplementary Fig. 8). Single crystals are assembled into heterostructures via a dry-transfer technique ${ }^{38}$. A transparent stack comprising a glass slide, a polydimethylsiloxane layer attached to the glass and polycarbonate (PC) as an external film is mounted on a micromanipulator positioned under an optical microscope with a temperature-controlled stage. After adjusting the alignment and bringing the transfer stack into contact with the exfoliated TMD flakes, these are picked up due to their higher adhesion to PC. The process is repeated for the hBN tunnel barrier. Finally, after aligning and bringing in contact hBN and TMD on PC with exfoliated SLG on $\mathrm{Si} / \mathrm{SiO}_{2}$, the temperature is raised to $\sim 100^{\circ} \mathrm{C}$, releasing the PC with hBN/TMD onto SLG. Then, the sample is soaked in chloroform to dissolve the PC film, leaving the SLG/hBN/TMD heterostructure on the $\mathrm{Si} / \mathrm{SiO}_{2}$ substrate. Finally, $\mathrm{Cr} / \mathrm{Au}(3 / 50 \mathrm{~nm})$ contacts both to SLG and TMD are patterned by e-beam lithography following a standard lift-off process. Heterostructures are characterized by Raman spectroscopy to ensure no degradation (see Supplementary Notes 1-3 for further details).

Confocal microscopy. PL and EL measurements are performed using a homebuilt confocal microscopy mounted on a three-axis stage (Physik Instrumente M-405DG) with a 5-cm travel range and 200-nm resolution for coarse alignment and a piezo scanning mirror (Physik Instrumente S-334) for high resolution raster scans (see Supplementary Fig.10 for a diagram of the optical set-up). PL and EL are collected using a $1.7-\mathrm{mm}$ working distance objective with a numerical aperture of 0.7 (Nikon S Plan Fluor $60 \times$ ) and detected on a fibre-coupled single-photoncounting module (PerkinElmer SPCM-AQRH). A variable-temperature helium flow cryostat (Oxford Instruments Microstat HiRes2) is used to perform PL and EL measurements. A controlled bias is applied to the QLED devices by a source measurement unit (Keithley 2,400) for EL experiments. Intensity correlations from the Hanbury Brown and Twiss interferometer are recorded with a time-to-digital converter (quTAU). A double-grating spectrometer (Princeton Instruments) is used for acquiring spectra. For PL measurements, the excitation laser $(700 / 520 \mathrm{~nm}$, Thorlabs MCLS1) is suppressed with a long pass filter (715 nm, Semrock FF01-715/ $550 \mathrm{~nm}$ Thorlabs FEL0550).

Data availability. The data that support the findings of this study are available from the corresponding authors upon request.

\section{References}

1. Yuan, Z. et al. Electrically driven single-photon source. Science 295, 102-105 (2002)

2. Mizuochi, N. et al. Electrically driven single-photon source at room temperature in diamond. Nat. Photonics 6, 299-303 (2012).

3. Lohrmann, A. et al. Single-photon emitting diode in silicon carbide. Nat. Commun. 6, 7783 (2015).

4. Nothaft, M. et al. Electrically driven photon antibunching from a single molecule at room temperature. Nat Commun. 3, 628 (2012)

5. Bonaccorso, F., Sun, Z., Hasan, T. \& Ferrari, A. C. Graphene photonics and optoelectronics. Nat. Photonics 4, 611-622 (2010).

6. Ferrari, A. C. et al. Science and technology roadmap for graphene, related twodimensional crystals, and hybrid systems. Nanoscale 7, 4598-4810 (2014).

7. Ye, Y. et al. Monolayer excitonic laser. Nat. Photonics 9, 733-737 (2015).

8. Sun, Z. et al. Graphene mode-locked ultrafast laser. ACS Nano 4, 803-810 (2010).

9. Liu, M. et al. A graphene-based broadband optical modulator. Nature 474, 64-67 (2011).

10. Phare, C. T., Daniel Lee, Y.-H., Cardenas, J. \& Lipson, M. Graphene electrooptic modulator with $30 \mathrm{GHz}$ bandwidth. Nat. Photonics 9, 511-514 (2015).

11. Koppens, F. H. L. et al. Photodetectors based on graphene, other twodimensional materials and hybrid systems. Nat. Nanotechnol. 9, 780-793 (2014).

12. Xia, F., Wang, H., Xiao, D., Dubey, M. \& Ramasubramaniam, A. Twodimensional material nanophotonics. Nat. Photonics 8, 899-907 (2014).

13. Srivastava, A. et al. Optically active quantum dots in monolayer $\mathrm{WSe}_{2}$. Nat. Nanotechnol. 10, 491-496 (2015).

14. He, Y.-M. et al. Single quantum emitters in monolayer semiconductors. Nat. Nanotechnol. 10, 497-502 (2015).

15. Koperski, M. et al. Single photon emitters in exfoliated $\mathrm{WSe}_{2}$ structures. Nat. Nanotechnol. 10, 503-506 (2015).
16. Chakraborty, C., Kinnischtzke, L., Goodfellow, K. M., Beams, R. \& Vamivakas, A. N. Voltage-controlled quantum light from an atomically thin semiconductor. Nat. Nanotechnol. 10, 507-511 (2015).

17. Tonndorf, P. et al. Single-photon emission from localized excitons in an atomically thin semiconductor. Optica 2, 347 (2015).

18. Tran, T. T., Bray, K., Ford, M. J., Toth, M. \& Aharonovich, I. Quantum emission from hexagonal boron nitride monolayers. Nat. Nanotechnol. 11, 37-41 (2015).

19. Yin, Z. et al. Single-layer $\mathrm{MoS}_{2}$ phototransistors. ACS Nano 6, 74-80 (2011).

20. Wang, H., Zhang, C., Chan, W., Tiwari, S. \& Rana, F. Ultrafast response of monolayer molybdenum disulfide photodetectors. Nat. Commun. 6, 8831 (2015).

21. Ross, J. S. et al. Electrically tunable excitonic light-emitting diodes based on monolayer WSe ${ }_{2}$ p-n junctions. Nat. Nanotechnol. 9, 268-272 (2014).

22. Baugher, B. W. H., Churchill, H. O. H., Yang, Y. \& Jarillo-Herrero, P. Optoelectronic devices based on electrically tunable $\mathrm{p}-\mathrm{n}$ diodes in a monolayer dichalcogenide. Nat. Nanotechnol. 9, 262-267 (2014).

23. Pospischil, A., Furchi, M. M. \& Mueller, T. Solar-energy conversion and light emission in an atomic monolayer p-n diode. Nat. Nanotechnol. 9, 257-261 (2014).

24. Withers, F. et al. Light-emitting diodes by band-structure engineering in van der Waals heterostructures. Nat. Mater. 14, 301-306 (2015).

25. Zhou, B. et al. Evolution of electronic structure in atomically thin sheets of WS and $\mathrm{WSe}_{2}$. ACS Nano 7, 791-797 (2013).

26. Jones, A. M. et al. Optical generation of excitonic valley coherence in monolayer $\mathrm{WSe}_{2}$. Nat. Nanotechnol. 8, 634-638 (2013).

27. Wang, G. et al. Valley dynamics probed through charged and neutral exciton emission in monolayer $\mathrm{WSe}_{2}$. Phys. Rev. B 90 (2014).

28. Kumar, S., Kaczmarczyk, A. \& Gerardot, B. D. Strain-induced spatial and spectral isolation of quantum emitters in mono- and bilayer $\mathrm{WSe}_{2}$. Nano Lett. 15, 7567-7573 (2015).

29. Hansom, J., Schulte, C. H. H., Matthiesen, C., Stanley, M. J. \& Atatüre, M. Frequency stabilization of the zero-phonon line of a quantum dot via phononassisted active feedback. Appl. Phys. Lett. 105, 172107 (2014).

30. Acosta, V. M. et al. Dynamic stabilization of the optical resonances of single nitrogen-vacancy centers in diamond. Phys. Rev. Lett. 108, 206401 (2012).

31. Eisaman, M. D., Fan, J., Migdall, A. \& Polyakov, S. V. Invited review article: single-photon sources and detectors. Rev. Sci. Instrum. 82, 071101 (2011).

32. Warburton, R. et al. Optical emission from a charge-tunable quantum ring Nature 405, 926-929 (2000).

33. Nowack, K. C., Koppens, F. H. L., Nazarov, Y. V. \& Vandersypen, L. M. K Coherent control of a single electron spin with electric fields. Science $\mathbf{3 1 8}$ 1430-1433 (2007).

34. Salter, C. L. et al. An entangled-light-emitting diode. Nature 465, 594-597 (2010).

35. Watanabe, K., Taniguchi, T. \& Kanda, H. Direct-bandgap properties and evidence for ultraviolet lasing of hexagonal boron nitride single crystal. Nat. Mater. 3, 404-409 (2004)

36. Casiraghi, C. et al. Rayleigh imaging of graphene and graphene layers. Nano Lett. 7, 2711-2717 (2007).

37. Ferrari, A. C. \& Basko, D. M. Raman spectroscopy as a versatile tool for studying the properties of graphene. Nat. Nanotechnol. 8, 235-246 (2013).

38. Bonaccorso, F. et al. Production and processing of graphene and $2 \mathrm{~d}$ crystals. Mater. Today 15, 564-589 (2012).

\section{Acknowledgements}

We acknowledge the financial support from the EU Graphene Flagship (no. 604391), ERC Grants Hetero2D and PHOENICS, EPSRC Grants EP/K01711X/1, EP/K017144/1, $\mathrm{EP} / \mathrm{N} 010345 / 1, \mathrm{EP} / \mathrm{M} 507799 / 1, \mathrm{EP} / \mathrm{L} 016087 / 1, \mathrm{EP} / \mathrm{M} 013243 / 1$ and the EPSRC Cambridge NanoDTC, EP/G037221/1. We are grateful to J. Barnes, C. Le Gall and H.S. Knowles for technical assistance.

\section{Author contributions}

M.A., A.C.F. and I.G. devised and managed the project. K.W. and T.T. provided highquality hBN material, M.B., X.C. and I.G. fabricated the devices, C.P.-B. and D.M.K. performed the optical measurements, assisted by M.B., X.C. and I.G., and analysed the data. M.B., C.P.-B., D.Y. and A.K.O. performed Raman, PL, optical contrasts and AFM measurements and analysis. J.B. developed part of the data acquisition software for the confocal microscope. All authors participated in the writing of the manuscript.

\section{Additional information}

Supplementary Information accompanies this paper at http://www.nature.com/ naturecommunications 
Competing financial interests: The authors declare no competing financial interests.

Reprints and permission information is available online at http://npg.nature.com/ reprintsandpermissions/

How to cite this article: Palacios-Berraquero, C. et al. Atomically thin

quantum light-emitting diodes. Nat. Commun. 7, 12978 doi: 10.1038/ncomms12978 (2016). (c) (i) This work is licensed under a Creative Commons Attribution 4.0 International License. The images or other third party material in this article are included in the article's Creative Commons license, unless indicated otherwise in the credit line; if the material is not included under the Creative Commons license, users will need to obtain permission from the license holder to reproduce the material. To view a copy of this license, visit http://creativecommons.org/licenses/by/4.0/

(C) The Author(s) 2016 